\title{
Lactation management centres: A step forward in successful breast feeding
}

\author{
S C Wickramasinghe
}

Sri Lanka Journal of Child Health, 2012; 41(2): 82-90

(Key words: Lactation management centre; breast feeding)

Breast milk (BM) is a unique product given to the human being by nature to fulfill all requirements of the offspring until it is mature enough to take adult food. Its uniqueness lies in the ability of the mother to produce milk which will vary in quantity, quality and consistency depending on the age of the baby, maturity and timing of the feed ${ }^{1}$. It has not been possible to achieve this with any other type of milk, even with state-of-the-art modifications using the most advanced technology. BM not only provides easily digestible and specifically needed amounts of nutrients, water, minerals and vitamins but also several other benefits to both mother and baby ${ }^{1}$.

\section{Benefits to the baby}

1. Reduces infections through "priming" of the baby's immune system.

- Specifically diarrhoea due to E coli, rotavirus, Shigella, campylobacter etc.

- Reduces incidence of respiratory tract infections

- Reduces late onset sepsis in low birth weight (LBW) babies

2. Effect on better neurodevelopment

3. Reduces risk of sudden infant death syndrome

4. Provides analgesia to the baby during painful procedures.

5. Long-term diseases like type I diabetes mellitus, hypercholesterolaemia, hypertension, obesity and asthma have been found to be less in babies who were exclusively breast fed during the first 6 months.

Benefits to the mother

1. Reduces postpartum bleeding

2. Reduces menstrual blood loss

3. Helps with child spacing attributable to lactational amenorrhoea

4. Reduces obesity

5. Reduces risk of breast cancer and ovarian cancer

6. Promotes bonding between mother and baby

7. Convenience of feeding the baby on demand irrespective of the time or place

8. Economical

${ }^{1}$ Consultant Paediatrician, Teaching Hospital, Kandy
Lactation reflexes ${ }^{2}$ are shown in Figure 1.

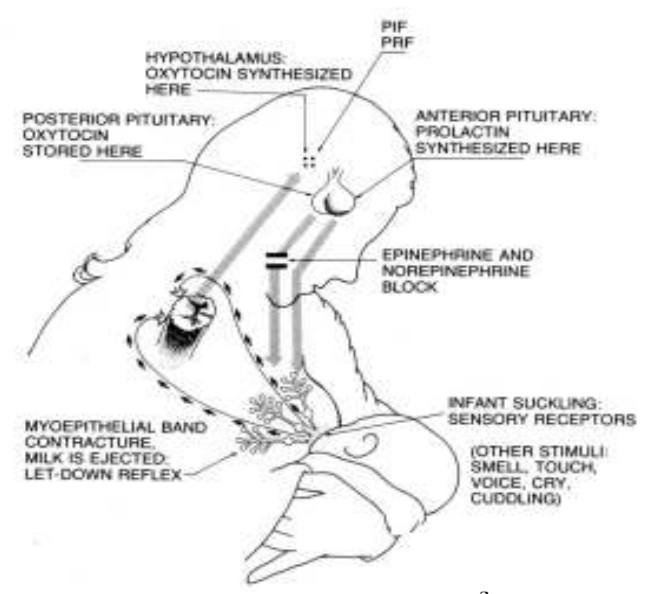

Figure 1: Lactation reflexes ${ }^{2}$

Factors affecting the oxytocin reflex $^{3}$ are shown in Figure 2.

\section{DXYTOCIN REFLEX}

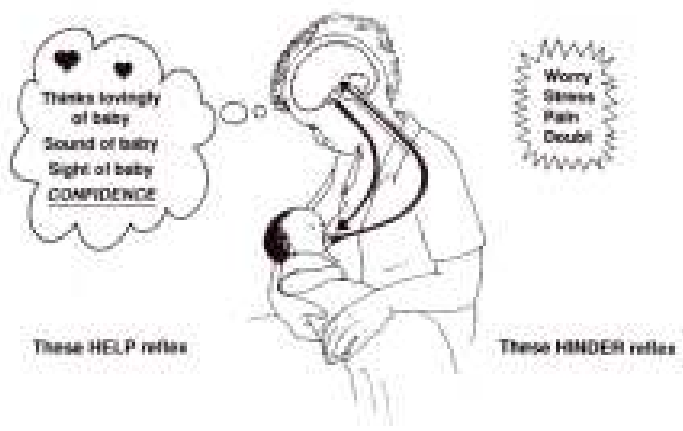

Figure 2: Helping and hindering oxytocin reflex ${ }^{3}$

Following are some current statistics related to neonates and breast feeding (BF) in Sri Lanka ${ }^{4,5,6}$ :

- Neonatal mortality rate: 5.9/1000 live births

- Infant mortality rate: $7.4 / 1000$ live births

- Percentage contribution of neonatal mortality rate to infant mortality rate $: 72 \%$

- Infection is one of the three common causes of morbidity and mortality in neonates.

- Percentage of babies breast fed within the $1 \mathrm{st}$ hour of age : $85 \%$ 
- Percentage of babies exclusively breast fed from $0-1$ month : $92.6 \%$

- Percentage of babies exclusively breast fed up to 3 months : $85.1 \%$

- Percentage of babies exclusively breast fed up to $4-5$ months : $53.5 \%$

In this scenario, it is pertinent to note that percentage reduction of infections in neonates by exclusive breast feeding (EBF) as a single intervention is known to be around $22 \%$.

Ninety percent of births in Sri Lanka take place in institutions. Mothers get satisfactory support and advice about EBF in most of the health facilities. As the figures given above indicate that the percentage of mothers who continued to breast feed exclusively go down with the age of the baby, it is important to look for possible causes of this phenomenon. Some possible reasons are:

- Issues with BF are not often identified as serious medical problems to get professional advice. Input given by elders, neighbours and friends play a key role.

- Spending time in busy clinics and queues is practically difficult for the mother and baby.

- Confusing advice is sometimes given by our own health staff.

- More nuclear families are seen in our society today. Mother may not get adequate support for BF when needed.

- Books and reading material do not provide solutions to every feeding problem.

- Above all, multi-national milk food companies are providing an easy alternative to a mother who encounters even a small problem with BF.

- Common myths such as frequent crying indicate inadequate amount of BM etc. which lead mothers to decide on adding artificial feeds to baby's diet.

If a baby with a feeding problem is admitted to a Special Care Baby Unit (SCBU) it may lead to many problems. However, this was the routine practice earlier. Some problems are:

- Mother and baby are separated. The basic and essential concepts of "rooming-in" and "bedding-in" cannot be implemented.

- Mother cannot perform demand feeding. Instead it will be interval feeding.

- It will have an impact on mother-baby bonding

- Practices like Kangaroo Mother Care (KMC), cannot be done.

- There is a significant risk of cross infections

- Cost per cot per day in SCBU is much more than that in a normal ward.
A satisfactory solution to these problems will be to have a separate area and dedicated staff to deal exclusively with the problems of BF.

Lactation management centre (LMC) is the brainchild of Dr. Anoma Jayathilake, then a Consultant Community Physician at the Family Health Bureau (FHB) and currently the National Professional Officer, World Health Organisation (WHO). However, the idea of having a separate area adjacent to SCBU, to keep both baby and mother together has been in the mind of a senior paediatrician, who was in charge of the SCBU at Castle Street Hospital for Women (CSHW), Dr.(Mrs) Devakanthi Gunarathne even though it became a reality long after her retirement due to the untiring efforts of Dr. A. Jayathilake. The concept of mother-baby units and LMCs was planned and received Health Ministry approval in 2007. Building, staff guidelines and equipment were all designed by a consultative committee of which the author of this article was a member. This was an important policy decision in a country like Sri Lanka where free health service is a significant burden on the economy. Few countries in the world have paid attention to this aspect and identified the need for a separate facility for mothers and babies who need special support for $\mathrm{BF}^{7}$. Although different terms have been used the basic functions of these units have been the same. Some examples in other countries are: Lactation Management Education Centre in Kenya and National Lactation Management Centre in Iran.

By definition, LMC is a part of the mother-baby unit and addresses mainly problems of $B F^{8}$. It functions as a day-care centre whereas babies who need overnight stay are admitted to mother-baby units. In Sri Lanka, the first LMC was started at CSHW, Colombo in the year 2000 and is running successfully to date. The second LMC was opened at General Hospital, Kandy (GHK) and thereafter many hospitals have installed such facilities.

Guidelines on construction of LMCs have been laid down by the FHB jointly with the Perinatal Society of Sri Lanka in collaboration with Central Engineering Consultancy Bureau (CECB) in 2007. This gives all details of facilities needed if a LMC is planned in a hospital. These standards are for a hospital with average monthly deliveries of 5001000. According to this document building and other instructions are given as follows:

- $\quad$ Space $20 \mathrm{~m}^{2}$

- Should be separated from duty station by a half wall up to a metre from the floor and a glass panel. 
- Should be separated from counselling room by a door, half wall up to a metre from the floor with a glass panel.

- Should accommodate five cots, one bed, 15 plastic arm chairs

- $\quad$ There should be stackable baby baskets to keep babies and wall lockers for mothers to keep their utensils (Can be plastic drawer type)

- There should be a sink with elbow operated tap.

- There should be a foldable nappy changing area

- Ceiling fans should be fixed.

- 24-hour water supply is mandatory.

- Soap racks to keep small pieces of soap for hand washing and towel dispensers

- Pictorial hand washing instructions should be displayed near sinks

- Ventilation: Cross ventilation and ceiling fans should be there. One or more rows of windows with aluminium frame at above 1.5 metre level $^{8}$.

- Ceiling should be washable / wet moppable. Asbestos free cement sheets should be used.

- Wall should be tiled up to $1.5 \mathrm{~m}$ from floor level. Ledges should be available to keep the items.

- Special type of grout should be used for tiles to prevent dust collection.

- Floor: Large tiles (2'x2') with matt finish should be used. Correct fixation is important to minimize the grooves for infection control.

- It must be in close location to postnatal wards, SCBU and main entrance of the hospital with easy access. Clear sign posting is essential. Dedicated elevator access should be available if located on a different floor.

- Doors: Ordinary type doors

- Lighting: Natural sunlight through windows is preferred.

- Lights should be fixed to the ceiling to minimize the dust collecting surfaces.

- Electricity: power outlets should be decided in consultation with the paediatrician.

Detailed description about the equipment, surgical inventory items, consumables, furniture etc. to mother-baby units are given in the same reference book. Selection of appropriate items should be made from these when a LMC is separately planned.

\section{Admission Criteria}

1. Mothers along with babies with feeding problems

2. Free from any other medical illness.
Other requirements for $L M C$

- It must be under direct supervision of the Consultant Neonatologist / Paediatrician in charge of SCBU.

- Medical officer coverage must be provided from SCBU. These doctors should preferably have had some training on BF.

- Nursing officers must be handpicked by the neonatologist, not on seniority but on good communication skills, kindness and patience with mothers and babies, keenness on promoting BF etc. They must be essentially given training on $\mathrm{BF}$, either a 40 hour $\mathrm{BF}$ course or Baby Friendly Hospital Initiative (BFHI). These training programmes are held from time to time by the FHB

- Best service coverage can be given if the number of nurses is as follows: One nurse/ maternity unit + one extra nurse.

- 1-2 minor staff workers or orderlies depending on the hospital size and work load at LMC.

- Opening times must be 7/7 days 7 am -5 pm

- There must be a telephone hotline for communication

- Mothers with problems in BF should be free to come without referral letter or appointments.

\section{Duties performed by the LMC Staff}

1. Consultant Neonatologist/Paediatrician

- Overall supervision

- Conducting/participating in in-service programmes on $\mathrm{BF}$

- $\quad$ Specialized opinion, if $\mathrm{MO}-\mathrm{SCBU}$ cannot handle the problem

- Special programmes - e.g. World BF day

- Educating the field staff on special occasions like monthly meetings, about the services provided by LMC.

2. $\mathrm{MO}-\mathrm{SCBU}$

- Attending medical problems when requested by nurses at $\mathrm{LMC}$

- Referring mothers and babies identified with feeding problems from postnatal wards, SCBU

- Helping at training programmes

3. Nursing officer - They have a major role to play

- $\quad$ Providing a relaxed, friendly environment.

- Identifying the problems: If there is concern of more than a feeding problem get medical attention.

- Doing daily rounds in postnatal wards: 1 nurse/ward

- $\quad$ Paying more attention to the mothers who were identified with feeding problems. 
- Visiting mothers who are at ICU's on request and help with expressing milk.

- Brief health messages to mothers in antenatal/postnatal wards during the routine rounds.

- Advise by telephone.

- Attending to the mothers who come to LMC, some of whom may be in-patients, some out-patients

- Contribution by means of talks on BF for antenatal health educational classes.

- Special day/half-day programmes organized to educate nursing officers and minor staff members working in other wards.

- Lectures/Practical sessions for nursing students and midwifery students as organized by Nurses Training Schools.

Maturation of oral feeding skills ${ }^{9}$ is shown in Figure 3.

\section{Maturation of oral feeding skills}

$<28$ Weeks

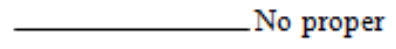
sucking efforts Often needs No proper motility of the gut full IV fluids

$28-31$ weeks

Sucking Bursts

No coordination between swallowing \& breathing

$\mathrm{OG} / \mathrm{NG} /$ feeds spoon/cup feeding

32-34 weeks

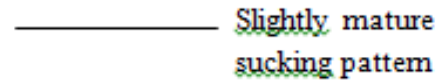
Co-ordination between breathing + swallowing begins

Mature sucking pattem Coordination between sucking + swallowing
Feeding by cup/spoon

Breast feeding

Breast feeding
$>34$ weeks

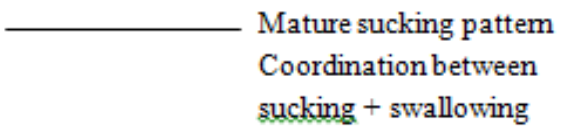

Figure 3: Maturation of oral feeding skills

\section{Common problems encountered at $\mathbf{L M C} \mathbf{C}^{10,11}$}

\section{Maternal Problems}

1. Flat/inverted nipples - antenatal diagnosis and attempts to correct is not often helpful. Instead,

- Build confidence

- Educate on the 'Syringe Method' to be used just before the feed

- Provide extra support

- Explain that baby suckles the breast not the nipple. Sucking helps to elongate the nipple

- Encourage frequent Skin To Skin (STS) contact

- Try different positions to hold the baby

2. Difficult breast - e.g. large breast

- Needs extra support

- Loosely tying up a rolled up nappy around the breast will help to improve the shape
3. Full breast

ภ

Not hard

No pain

Milk flowing

No fever<smiles>[C]=[Ge]</smiles>

Allow more frequent sucking
Engorged breast

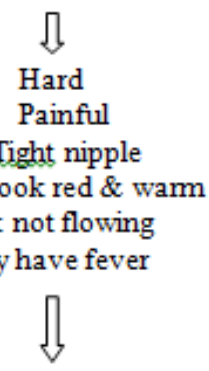

Correct techniques of position and attachment Warm compresses and express by hand/pump Cold compresses after feeding 
3. Blocked duct and mastitis due to poor drainage of part or whole of the breast can occur due to stress, over work and trauma to the breast.

- Encourage frequent feeding

- Relax

- Gentle massage towards the nipple

- Warm compresses

- Paracetamol as an analgesic when necessary

- Cloxacillin for $7-10$ days, if indicated

4. Cracked or sore nipple;

- Correct attachment

- Avoid frequent washing of nipple with soap and water

- Apply hind milk and rub around the nipple after each feed

5. Candidiasis of breast and oral thrush in baby

- For baby: crushed nystatin tablets or mouth paint/oral gel 6 hourly for 7 days

- $\quad$ For mother: Topical antifungal as above 6 hourly for 7 days after baby gets a feed.

\section{Common Problems with babies}

1. Poor weight gain: Correct measurement of weight is essential for proper evaluation

- Correct techniques of feeding

- Top up feeding of EBM by spoon/cup

- Medical advice to exclude an organic cause.

2. Excessive crying: Exclude other causes for crying and confirm that it's due to a feeding problem

- Exclude medical causes e.g. Infantile colic, ear infection, CNS infection

- Mother's food - Cow's milk, peanuts etc.

- Drugs taken by mother - Coffee, Cola drinks

- "High need" babies

3. Excessive sleepiness

- Exclude problems like

$$
\begin{array}{ll}
\circ & \text { Hypothyroidism } \\
\circ & \text { Chromosomal Diseases } \\
\circ & \text { Maternal drugs }
\end{array}
$$

- Correct techniques of feeding

\section{Common Procedures performed at $\mathrm{LMC}^{12}$}

1. Weighing the babies and monitoring growth after correcting the feeding techniques

2. Correcting positioning and attachment

3. Helping/Teaching mothers how to express and spoon or cup feed the baby.

4. Back massage/Let down relax

5. Hot and cold compresses to breasts when engorged.
6. Using "Syringe Method" for mothers with inverted/flat nipples.

7. General counselling/reassuring and encouraging the mother

Allowing them to share experiences with each other, clearing their doubts, myths etc.

When a mother has inverted or flat nipples "Syringe Method"3 is a useful technique just before a breast feed (Figure 4).

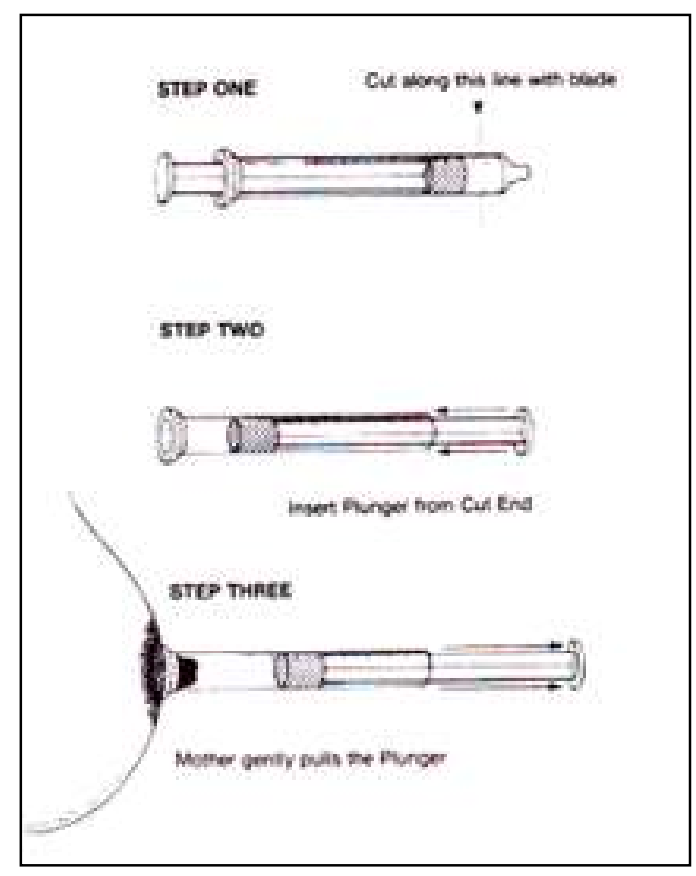

Figure 4: Preparing and using a syringe for treatment of inverted nipples

\section{Accurate measurement of weight}

- Is an essential procedure at the LMC

- Electronic scale that is accurate to the nearest $2-3 \mathrm{gm}$ is essential

- Weigh before and after a feed under exactly same circumstances (e.g. Do not change the diaper in between weighing)

- Baby does not need to be nude during weighing

- 1 gm of weight gain equals $1 \mathrm{ml}$ of milk intake

- If the baby in connected to a monitor, disconnect the wires and lay them on top of the baby rather than allowing them to hang over the side of the scale.

Monitoring adequacy of breast feeding ${ }^{13}$ is shown in Figure 5. 
Monitoring adequacy of breast feeding

1. Observe

Attachment \& positioning, evidence of nipple/

breast problems, sucking technique

2. Instruction to mother

Is the baby having at least 6 wet nappies/day after the $3 \mathrm{rd}$ day of life?

Is the baby having at least 3 stools/day after the 3 rd day of life?

Is he taking $8-12$ feeds/day (every $1 \frac{1 / 2}{2}-3$ hours)?

3. Expected growth

Is he sucking $10-20 \mathrm{~min} /$ breast each feed?

Weight loss $<10 \%$ of birth weight

No further weight loss after day 3-5

Regains birth weight by day $7-14$

Steady weight gain 20-30 g/day after the age of 1 week

LBW babies - $15-20 \mathrm{~g} / \mathrm{kg} / \mathrm{d}$ until they reach $2.5 \mathrm{~kg}$; thereafter $20-30 \mathrm{~g} /$ day is a dequate.

Figure 5: Monitoring adequacy of breast feeding

Back massage to stimulate the oxytocin $\operatorname{reflex}^{3}$

(Figures 6 \& 7)

- Helps the mother psychologically

- Builds her confidence

- Tries to reduce pain or anxiety

- Helps her to have good thoughts about her baby

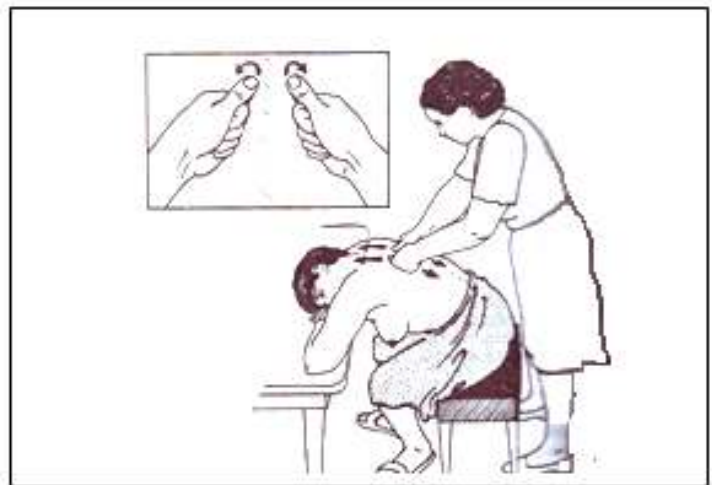

Figure 6: Back rub to stimulate oxytocin reflex

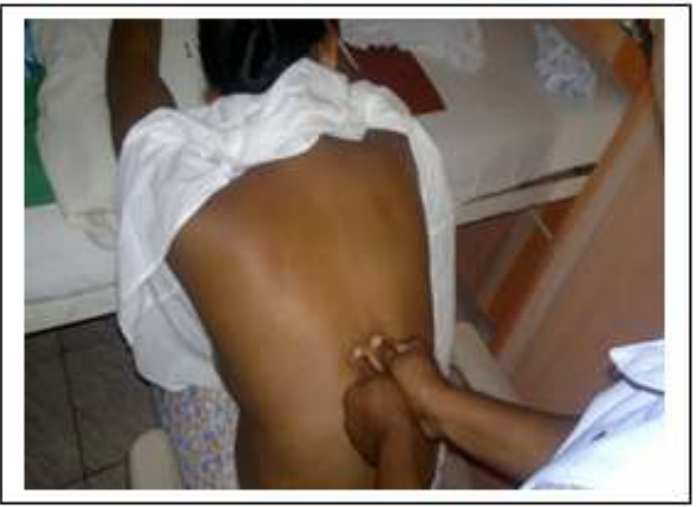

Figure 7: Back rub in practice
Help her practically

- Sit quietly and in privacy

- If possible, keep the baby close by

- Help the mother to take a warm drink

- Warm the breasts with a wet towel

- Stimulate her nipples by touching

- Stroke the breast gently with fingers or a comb

Mother sits, leaning forward, arms folded on a table, head resting on arms. Breasts must hang loose, unclothed. Helper rubs on either side of her spine using her fists, thumbs pointing forward, with small circular movements. Massaging should be done from the neck to the tip of the shoulder blade, up and down for few minutes.

Steps of paladai / feeding ${ }^{11,14}$ (Figure 8)

- Wash hands

- Place the infant upright on mother's lap

- Napkin around the neck

- Fill the cup/paladai a little short of the rim

- Place it at the lips and gently tilt to the top of the lip

- Let the baby actively swallow

- Gentle stimulation will help if the baby is sleepy

- Calculate the volume needed for the age and weight and volume taken by the baby

- Wash the cup with soap and water, boil for 20 minutes to sterilize before the next feed 


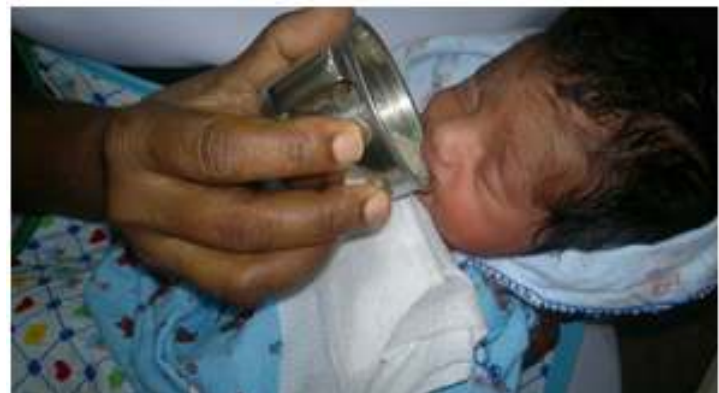

Figure 8: Cup feeding

Steps of expression of BM (Figure 9)

- Wash hands

- Clean wide mouthed container

- Massage the breast for 5-10min (Use the fingers or knuckles of the fist in a circular motion towards the nipple.

- Place thumb above the nipple and areola, index finger below the areola opposite the thumb

- Support the breast with other fingers

- Ask her to press her thumb and index finger towards the chest wall

- Press the breast behind the nipple and areola between thumb and index finger

- Press and release, press and release right round the areola

- It should not hurt if the technique is correct.

- Change the sides when milk flow slows down.

- Avoid rubbing the skin or squeezing the nipples.

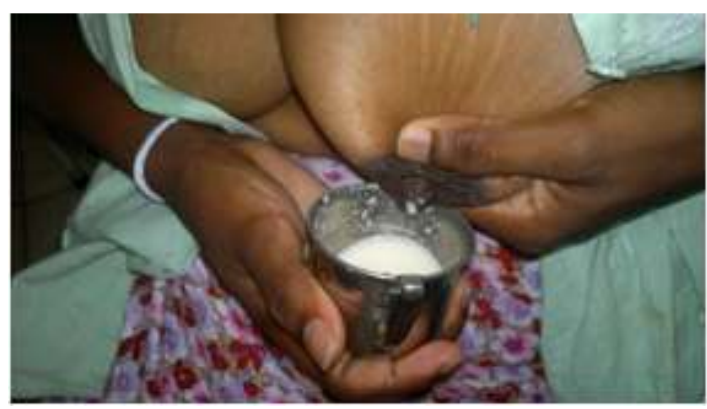

Figure 9: Expression of breast milk

Advice on storage of $\mathrm{BM}$

A container with a lid which has been boiled to sterilize for 10 minutes is suitable to store the EBM.

- Can be kept safely for 6 hours at room temperature

- In the fridge -24 hours

- In the freezer compartment -3 months

Milk which has been refrigerated must be kept in a warm water bath and adequately warmed for a few minutes before feeding,

\section{Experience at LMC, GHK}

GHK is the second biggest hospital in the country. It has 78 wards and 13 special units with total bed strength of 2291. There are 3 obstetric units (270 beds), 3 labour rooms and 4 Consultant Obstetricians. There are over 15,000 deliveries per year. GHK was declared a Baby Friendly Hospital in 1993. LMC was started in June 2006 (second in the country) (Figure 10)

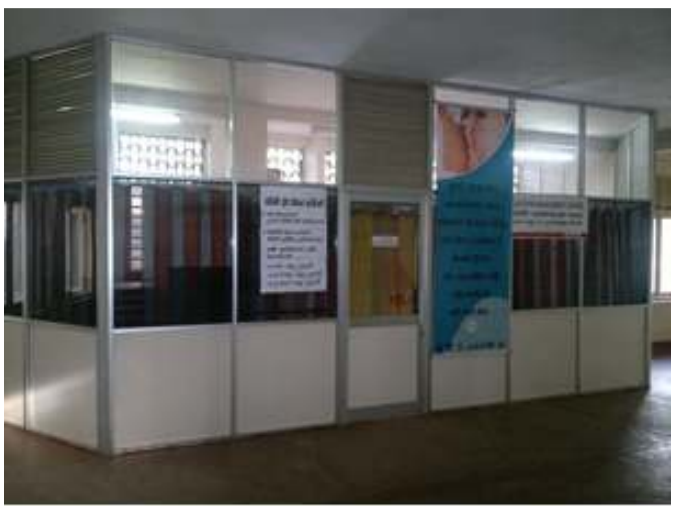

Figure 10: LMC at Teaching Hospital, Kandy

There is a day area (a small partition from a corridor) which is open 7 days a week from 7 a.m. to 5 p.m. The Staff consists of the Consultant Paediatrician in charge of SCBU, 4 staff nurses (all trained in BFHI course), 2 minor staff and a medical officer cover from SCBU staff.

There is open access to all mothers (no referral letters needed). There are referrals from OPD/ clinics/SCBU/postnatal wards/paediatric medical and surgical wards. After the first visit reviews are arranged by nurses. There is no age limit for babies (usually under 1 year)

\section{How to get information about LMC}

1. Rubber stamp with advice and contact number on CHDR (Figures $11 \& 11$ )

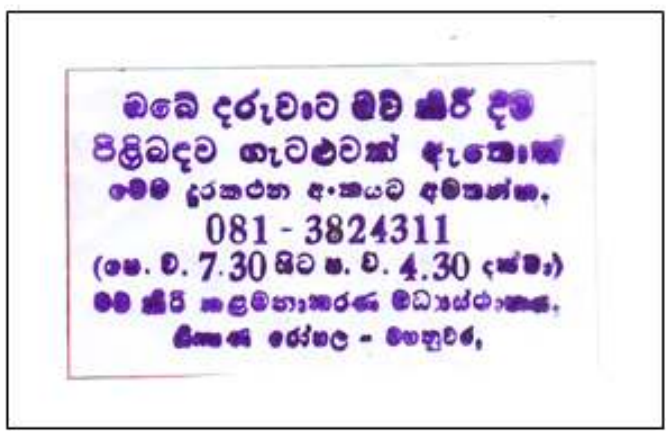

Figure 11: Information about LMC on a rubber stamp 


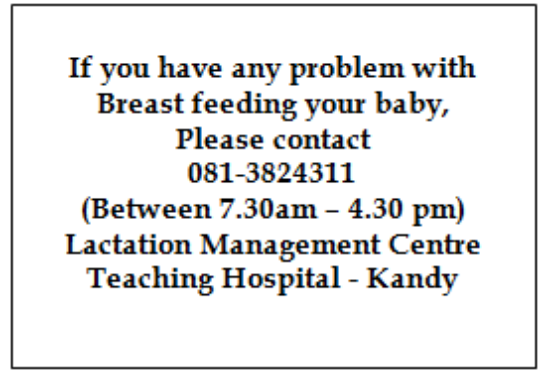

Figure 11 a: English translation

2. Doctors and Consultant Paediatrician

3. Field midwives and medical officers of health

4. Other hospital staff

\section{Duties of LMC Staff}

- Daily ward rounds in postnatal wards

- Special attention to mothers who had feeding problems in the previous night ( Notification book is maintained)

- Visiting mothers' in ICU/CCU on request

- Attending mothers' who directly come to LMC

- Answering telephone inquiries and advice

- Minor staff members sent to ICU/CCU to bring EBM for the baby

- In service programs to train hospital staff

- Educational programs to parents-to-be at antenatal clinics

- Maintenance of registers with statistics, sending monthly returns to FHB

\section{Services provided}

- Offers a relaxed environment

- Monitoring weight gain

- Correcting techniques of breast feeding

- Advice on special problems e.g. sore nipple

- Teaching mothers on spoon feeding, expressing milk, back massage, Kangaroo Mother Care

- $\quad$ Advice on top up feeding when necessary - in case of artificial feeding advice from Senior $\mathrm{MO} /$ Paediatrician is obtained.

\section{Statistics}

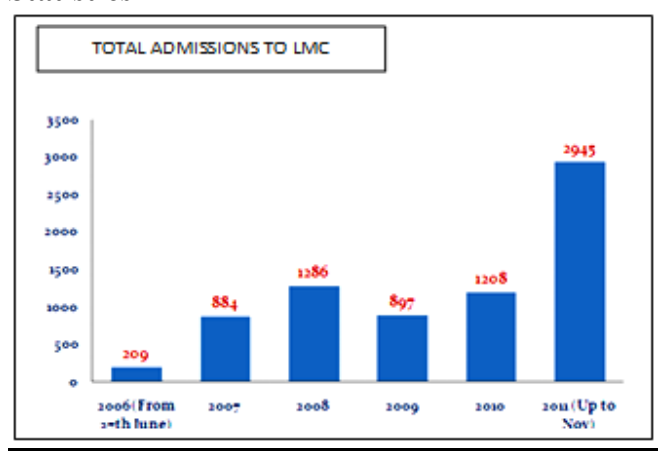

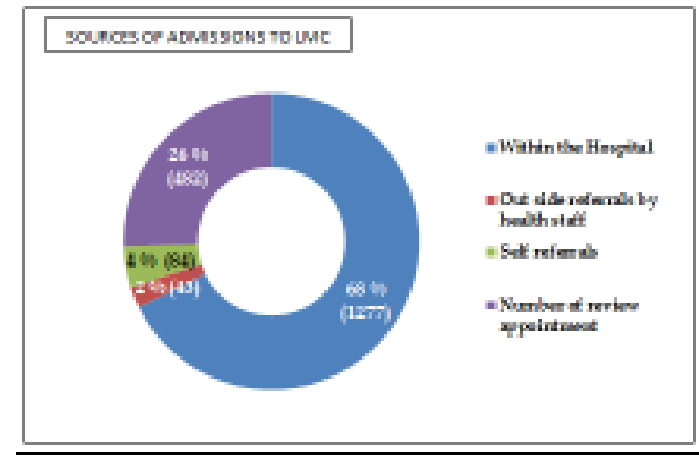

Figure 13: Sources of admissions to LMC

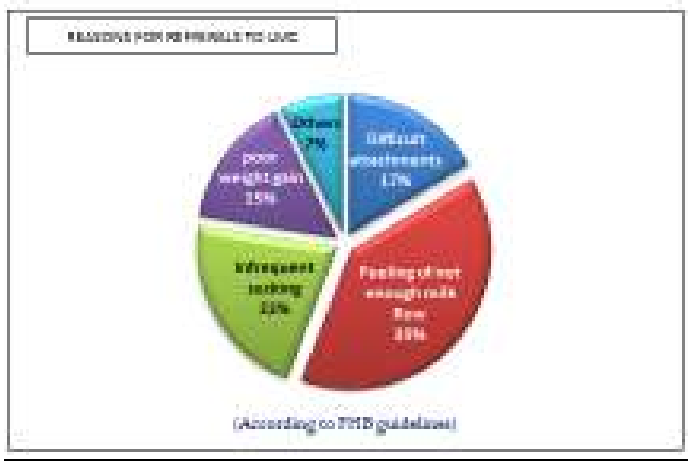

Figure 14: Reasons for Referrals to LMC

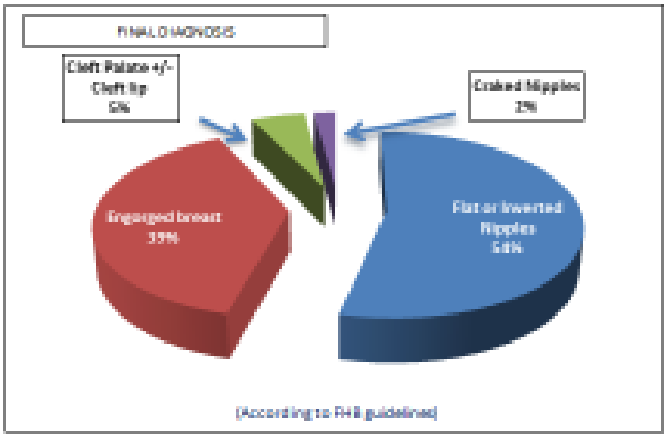

Figure 15: Final diagnosis of LMC referrals

Monthly feed-back is expected to be sent from all the LMC's around the country to FHB in a standard form which is shown in figure 16. This has been started since 2008 and is in the process of being regularized gradually.

\section{Final remarks}

The concept of starting LMCs must be introduced to every health facility in the country and the paediatricians have a key role to play in the maintenance of services and standards ${ }^{15}$.

Figure 12: Total admissions to LMC 


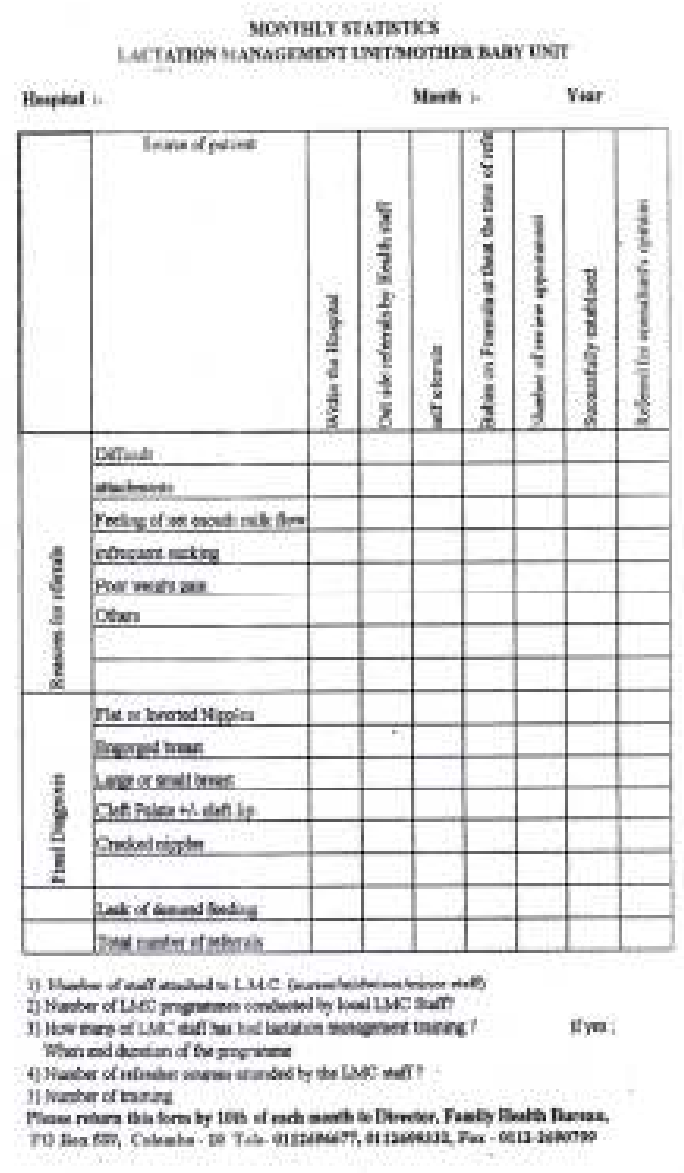

Figure 16: Monthly returns form from LMC to FHB

\section{References}

1. Sourabh Dutta PK, editor. Composition of various types of milk, Clinical benefits of human milk. In Neonatal Nutrition. Chandigarh; 2008. p. 6-12.

2. Hervada A. Physiology of human lactation. Paediatrics and Nutrition Review. 1982: p. 7.

3. Expressing breast milk. In breastfeeding counselling: A training course. Geneva: World Health Organization; 1993. p. 14,70.

4. Department of Census and Statistics - Sri Lanka. [Online].; 2010 [cited 2012 January 30. Available from: http://www.statistics.gov.lk/Newsletter/vital\% 20statistics.pdf.
5. Sri Lanka Demographic and Health Survey 2006 -2007. Colombo: Department of Census and Statistics, Ministry of Health Care and Nutrition; 2009.

6. Karen M. Edmond CZMAQSAESOABRK. Delayed breastfeeding initiation increases risk of neonatal mortality. Paediatrics. 2006.

7. Hossain M A MIHABSMIB. Activities of LMC of Rajshatri. TAJ. 2005 December; XVIII (2).

8. Building and Other Guide Lines for Neonatal intensive care units, Special care baby units, Mother baby centres. Colombo: Family Health Bureau , Ministry of Health Care and Nutrition, Perinatal Society of Sri Lanka, Centra- Engineering Consultancy Bureau; 2007.

9. Omari TI RC. Fetal and neonatal physiology. 2nd ed. Philadelphia: W B Saunders Co; 1998.

10. Trainer Guide On Perinatal Care Colombo: Perinatal Society of Sri Lanka, Family Health Bureau; 2004.

11. Deorari AK, editor. Skills related to feeding. In Practical procedures for the Newborn Nursery ( A Manual for Physicians \& Nurses). New Delhi; 2010. p. 101-112.

12. Feeding and fluid management. In integrated management of pregnancy and childbirth managing newborn problems. Geneva: WHO, UNFPA, UNICEF.

13. Jill Comphell CCMMW, editor. Infant Nutrition. In Paediatric Nutrition Reference Guide. 7th ed.: Texas Children's Hospital; 2005. p. 22-23.

14. All India Institute of Medical Sciences (AIIMS). Protocols in neonatology Deorani AK, editor. New Delhi.

15. Report of the external review of Maternal and Newborn Health Sri Lanka. Colombo: Ministry of Health Care \& Nutrition; 2007. 